\title{
Using animal models of enriched environments to inform research on sensory integration intervention for the rehabilitation of neurodevelopmental disorders
}

\author{
Stacey Reynolds • Shelly J. Lane $\cdot$ Lorie Richards
}

Received: 9 March 2010 / Accepted: 28 May 2010 /Published online: 12 June 2010

(C) Springer Science+Business Media, LLC 2010

\begin{abstract}
The field of behavioral neuroscience has been successful in using an animal model of enriched environments for over five decades to measure the rehabilitative and preventative effects of sensory, cognitive and motor stimulation in animal models. Several key principles of enriched environments match those used in sensory integration therapy, a treatment used for children with neurodevelopmental disorders. This paper reviews the paradigm of environmental enrichment, compares animal models of enriched environments to principles of sensory integration treatment, and discusses applications for the rehabilitation of neurodevelopmental disorders. Based on this review, the essential features in the enriched environment paradigm which should be included in sensory integration treatment are multiple sensory experiences, novelty in the environment, and active engagement in challenging cognitive, sensory, and motor tasks. Use of sensory integration treatment may be most applicable for children with anxiety, hypersensitivity, repetitive behaviors or heightened levels of stress. Additionally, individuals with deficits in social behavior,
\end{abstract}

S. Reynolds $(\bowtie) \cdot$ S. J. Lane

Department of Occupational Therapy,

School of Allied Health Professions,

Virginia Commonwealth University,

Box 980008, Richmond, VA 23298, USA

e-mail: reynoldsse3@vcu.edu

\section{S. Reynolds $\cdot$ L. Richards}

Department of Occupational Therapy,

School of Public Health and Health Professions,

University of Florida,

Box 100164, Gainesville, FL 32610, USA social participation, or impairments in learning and memory may show gains with this type of treatment.

Keywords Enriched environment · Sensory integration . Neural plasticity $\cdot$ Autism $\cdot$ Rehabilitation

Atypical patterns of sensory and motor behavior are present in a number of developmental diagnostic conditions including autism spectrum disorders (ASD). Sensory Integration treatment, aimed at ameliorating these behaviors, is one of the most common rehabilitation techniques sought out by parents of children with these disorders (Green et al. 2006). The theoretical framework underlying this treatment strategy suggests that actively engaging children in complex and meaningful sensory and motor activities in an opportunityrich environment can produce meaningful changes in attention and arousal regulation, motor planning and coordination, and social interaction and play abilities. Evidence for the effectiveness of sensory integration treatment is emerging (Fazlioglu and Baran 2008; Miller et al. 2007; Schaaf and Nightlinger 2007; Smith et al. 2005), but is plagued with methodological issues spanning from what constitutes sensory integration treatment to what outcomes are expected from this type of intervention (Parham et al. 2007; Williames and Erdie-Lalena 2009). At the same time, the field of behavioral neuroscience has been successful in using a paradigm of enriched environments for over five decades to measure the rehabilitative and preventative effects of sensory, cognitive and motor stimulation in animal models. Much of this work has been done with rats and mice, and this rodent work will be the focus of this paper. While extreme caution must be employed when 
comparing human and animal models, it is worth considering how successful and related animal paradigms can better inform selection of treatment parameters and outcome measures in human studies. The aims of this paper are to review the paradigm of environmental enrichment, compare essential features of animal models of enriched environments to principles of sensory integration treatment, and discuss applications for the rehabilitation of neurodevelopmental disorders.

\section{Enriched environments}

The concept of enriched environments was first described by Hebb (1947) who noted qualitative differences in behavior between the rats he brought home for his children to play with, and the rats who were cage-kept in the laboratory. It was not until the early 1960s that biochemical and structural changes were found in the brains of rats exposed to enriched laboratory environments, providing evidence that changes in the complexity of the environment and interaction in that environment can have a direct impact on the central nervous system (Krech et al. 1960; Rosenzweig et al. 1962). Since that time, numerous studies have used paradigms of enriched environments to study the mechanisms of experiencedependent plasticity in the central nervous systems of rats and other animals (for a review see Nithianantharajah and Hannan 2006).

Environmental enrichment involves changes to an animal's home cage or secondary exploratory area which provide enhanced sensory, motor, cognitive and potentially social opportunities. These enhancements are relative to standard housing conditions which generally entail a cage with bedding and access to food and water (Nithianantharajah and Hannan 2006). While different labs have employed different protocols, an enriched environment is often a large space (relative to the home cage) which allows exploration and the introduction of a variety of objects. These objects, varying in shape, size, weight, smell and texture, may include tubes, balance platforms, climbing apparatuses, balls, or running wheels which are generally changed out or manipulated in some way on a scheduled basis to maintain the concept of novelty and complexity in the environment. Nithianantharajah \& Hannan, suggest that the key feature of environmental enrichment is the aspect of environmental complexity, provided through a range of opportunities for stimulation in areas of visual, somatosensory, and olfactory systems. The changes noted following environmental enrichment are often described as experience dependent, indicating that they result from active interaction between the animal and the affordances available in the environment.

\section{Sensory integration treatment}

Sensory Integration (SI) treatment was originally described by Ayres who noted that "the child must participate actively with the environment to improve the organization of his nervous system...equipment used in sensory integrative therapy is designed to entice the child into activities that provide sensations that tend to organize young human brains" (1998, p. 142). Thus from its inception, sensory integration treatment has been conceptualized as an active process in which the child, guided by a trained therapist, engages with the physical environment to promote neurological change. By definition, sensory integration intervention provides the child with environmental complexity and the opportunity to experience a variety of sensory inputs. Further, because sensory integration intervention is designed on the basis of individual child needs, creating for each child an environment that entices engagement and interaction, gains made by the child are experience dependent. As such, sensory integration intervention embraces the key features of environmental enrichment presented above.

In the last decade, however, the growing popularity and marketability of sensory-based therapeutics has led to the emergence of a variety of programs and products claiming to be sensory integration. These programs, which may include children passively listening to music, repeating computerized motor patterns, or wearing special garments, often lack the core principles originally described by Ayres. In an attempt to identify and characterize fidelity to sensory integration treatment, Parham and colleagues (2007) reviewed key literature on the theoretical and pragmatic guidelines for SI intervention. From this review, core process elements were identified which must be present in order for an intervention to be considered sensory integration. These core process elements align with the model of enriched environments, specifically the importance of providing multiple sensory opportunities/ stimuli, providing increasing challenges, freedom to explore and choose activities, and providing equipment and arranging the room to facilitate engagement (Parham et al. 2007; Parham et al., in press). What appears to be unique about SI treatment, when compared to the paradigm of environmental enrichment, is the role of the therapist in facilitating interaction and motivation, supporting optimal arousal, and working beyond the therapy session with families and teachers (Parham et al., in press). Parham and colleagues (2007) identified these therapist-child interaction features as fostering of a therapeutic alliance between therapist and child, and deemed them a critical aspect of the core elements of Ayres sensory integration intervention. Table 1 presents a comparison of essential features of Ayresbased SI clinical treatment and the animal model of environmental enrichment. 


\section{Neurological outcomes of enriched environments}

Scientists have proposed that environmental enrichment, that is animals engaging with novel and complex multisensory environments, should result in enhancement in neural plasticity. Neural plasticity is the ability of the nervous system to change in response to experience or the environment (Breedlove et al. 2007). While neural plasticity is is a fundamental property of the brain, present with or without enrichment, it is believed that sensory enrichment can be powerful in driving meaningful and functional changes in the cortex through plastic mechanisms. These changes can be measured in a variety of ways both in neurons themselves and in cells that support neuronal growth and function.

One way enriched environments have been shown to change brain structures is through changes in dendritic branching and synaptogenesis. Because enriched environments facilitate performance of new and more integrated sensory and motor patterns, researchers hypothesized that both the number of dendrites and the number of dendritic connections should increase in response to this paradigm. Research supporting this hypothesis abounds, with specific changes noted in the visual, auditory and somatosensory cortexes and in the hippocampus (Bose et al. 2010; Connor et al. 1982; Faherty et al. 2003; Greenough and Volkmar
1973; Leggio et al 2005; Turner and Greenough 1985). Additional dendritic enhancement has been noted in areas of the cerebellum and motor cortex (Greenough et al. 1986; Floeter and Greenough 1979; Turner et al. 2003).

Further, new experiences, facilitated by the enriched environment, have been shown to generate new neurons. Neurogenesis has been explicitly found in areas of the hippocampus following environmental enrichment paradigms (Kempermann and Gage 2000; Brown et al. 2003) and these changes have been directly correlated with improvements in learning and memory (Bruel-Jungerman et al. 2005). Additional evidence of cell proliferation has been demonstrated in the amygdala, a key emotion-related structure in the brain (Okuda et al. 2009). This growth of new cells has been demonstrated throughout the lifespan, and experiencedependent plasticity appears to serve as a protective factor against diseases such as Alzheimer's and Parkinson's (Faherty et al. 2005; Herring et al. 2009; Savioz et al. 2009).

Plasticity has also been reported in non-neuronal cells such as astrocytes and oligodendrocyptes, glial cells present throughout the brain (for a review see Dong and Greenough 2004). These cells play a key role in support and protection of neurons, specifically in myelination of axons and anchoring neurons to their blood supply. Recent work suggests a role for glial cells in neurotransmitter availability for synaptic plasticity (Bezzi et al. 2004). These enhancements in gliogenesis

Table 1 Comparison of essential features of SI and enriched environment models

\begin{tabular}{|c|c|c|}
\hline Essential features & Enriched environments & SI intervention \\
\hline Sensory Experiences & $\begin{array}{l}\text { Large cage to explore, equipment to climb, balance, } \\
\text { smell, touch, and push. }\end{array}$ & $\begin{array}{l}\text { Large therapy space with points of suspension for swings. } \\
\text { Ramps, tunnels, balls, cushions, climbing and jumping } \\
\text { devises. }\end{array}$ \\
\hline Structural Features & $\begin{array}{l}\text { Adequate space and multi-sensory equipment must be } \\
\text { present and available during "treatment" condition }\end{array}$ & $\begin{array}{l}\text { Adequate space and multi-sensory equipment is necessary. } \\
\text { Also, therapist must be present and have adequate } \\
\text { training/qualifications. }\end{array}$ \\
\hline Novelty & $\begin{array}{l}\text { Experimenter changes the material presented in cage on } \\
\text { scheduled basis. }\end{array}$ & $\begin{array}{l}\text { Variety of materials available. Therapist's role to facilitate } \\
\text { more challenging or imaginative play activities and } \\
\text { change materials as deemed appropriate on a child by } \\
\text { child and session by session basis. }\end{array}$ \\
\hline Challenge & $\begin{array}{l}\text { Complexity of environment provides opportunities for } \\
\text { more complex motor patterns and integrative } \\
\text { experiences. }\end{array}$ & $\begin{array}{l}\text { The environment itself should afford sensory-motor chal- } \\
\text { lenges, but it is the role of the therapist to facilitate the } \\
\text { "just-right challenge". }\end{array}$ \\
\hline Active Engagement & $\begin{array}{l}\text { To benefit from the enriched environment, animals must } \\
\text { actively engage with the materials in the environment. }\end{array}$ & $\begin{array}{l}\text { Child collaborates with therapists on activity choice and is } \\
\text { actively engaged in planning and executing sensory and } \\
\text { motor activities. }\end{array}$ \\
\hline Play/Enjoyment & $\begin{array}{l}\text { Sensory and motor experiences are generally enjoyable } \\
\text { and encourage engagement (sensory stimuli presented is } \\
\text { not noxious or aversive) }\end{array}$ & $\begin{array}{l}\text { Therapist creates a context of play- facilitates child's own } \\
\text { social, motor, imaginative or object play. }\end{array}$ \\
\hline Social & $\begin{array}{l}\text { Sometimes multiple animals are introduced to the } \\
\text { enriched environment simultaneously to facilitate social } \\
\text { interaction. }\end{array}$ & $\begin{array}{l}\text { Primary social relationship is between therapist and child. } \\
\text { Social interaction between children may occur but this is } \\
\text { not identified as a core element of SI treatment. }\end{array}$ \\
\hline Safety & $\begin{array}{l}\text { When social interactions occur, generally animals are of } \\
\text { the same size, and "bully" animals are not introduced } \\
\text { into the cage. }\end{array}$ & $\begin{array}{l}\text { Therapist ensures physical safety through placement of } \\
\text { equipment and maintaining proximity to child. Emotional } \\
\text { safety in the form of sustaining optimal levels of arousal. }\end{array}$ \\
\hline
\end{tabular}


and neurogenesis along with changes in structural connectivity are noted by Dong and Greenough (2004) to be important considerations for conditions such as autism, epilepsy, and Fragile X syndrome which have underlying abnormalities in both neurons and glia.

\section{Functional outcomes of enriched environments}

Functional outcomes of enriched environments have been measured based on 1) proposed neurological changes, and 2) proposed etiology or underlying symptomatology in the animal models. Globally, functional changes have been noted in the areas of motor performance, learning and memory, social-emotional behavior and sensory processing in animal models of neurodevelopmental disorders.

Autism is a neurodevelopmental condition with a strong genetic component (Bohm and Stewart 2009; Folstein, and Rosen-Sheidley 2001; Santangelo and Tsatsanis 2005), characterized by atypical social behaviors, restricted interests and stereotyped behaviors, and impairments in language and communication (American Psychiatric Association [APA], 2000). Atypical patterns of sensory processing have been widely reported in this population (Baranek et al. 2006; Leekham et al. 2006; Tomchek and Dunn 2007). The development of animal models for investigation of autism has guided understanding of both behaviors associated with autism, and potential interventions. In one model, rats are injected with valproic acid (VPA) on day 12.5 of gestation; the behaviors that unfold with development simulate symptoms of autism. These animals demonstrate lower sensitivity to pain, higher sensitivity to non-painful stimuli (hypersensitivity), locomotor and repetitive/stereotypic-like hyperactivity, lower exploratory activity, decreased number of social behaviors and an increased latency to social behaviors (Schneider and Przewlocki 2005). At the cellular level, diminished acoustic prepulse inhibition, a measure of cellular adaptation to sensory input, is also characteristic of VPA injected rats. Interestingly, exposure of VPA treated animals to environmental enrichment reverses many of these effects including normalizing sensitivity to pain, reducing hypersensitivity to non-painful stimuli, reducing locomotor and repetitive stereotypic-like activity, enhancing exploratory activity, decreasing anxiety, increasing the number of social behaviors, shortening the latency to social explorations, and strengthening acoustic prepulse inhibition (Schneider et al. 2006).

Fragile- $\mathrm{X}$ syndrome is a genetic disorder considered to be part of the autism spectrum and associated with atypical neural development. Symptoms of Fragile-X, including intellectual impairment, hypersensitivity, repetitive behaviors, and impairments in social interactions, are attributed to a failure to express the FMR1 gene. Animal models of
Fragile-X involve manipulating or knocking out (KO) the gene attributed to the production of the FMR1protein to mimic the symptomatology expressed in the human population. Using a mouse model of Fragile X syndrome, Restivo and colleagues (2005) demonstrated that engagement in enriched environments largely reduced anxiety and increased exploratory behavior. Signs of hyperactivity, a hallmark of both human Fragile-X and the animal KO model, did not improve with the enrichment paradigm.

Rett Syndrome is another genetic disorder classified as an autism spectrum disorder. It is caused by mutations in an $\mathrm{X}$-linked gene for methyl CpG-binding protein 2 (MeCP2). $\mathrm{MeCP} 2$ is believed to play an important role in controlling neuronal maturation in areas of the cortex, hippocampus, cerebellum, striatum, and thalamus (Stearns et al. 2007). In the human population, Rett Syndrome is characterized by a pattern of apparently typical development for the first few years of life, followed by a progressive decline in motor and cognitive functions. Stereoptyed behaviors such as excessive hand wringing are also frequently observed (Christodoulou and Williamson 2006). Several mouse models of Rett Syndrome have been developed (e.g. $\mathrm{MeCp} 2^{\text {tm1Tam }}$ and $\mathrm{MeCp} 2^{1 \text { lox }}$ ) which have been shown to exhibit motor, cognitive, and behavioral features consistent with the human disorder (Stearns et al. 2007). Environmental enrichment has been shown to improve motor coordination, motor learning, and/or locomotor activity in three different MeCP2 mutant strains (Kondo et al. 2008; Lonetti et al. 2010; Nag et al. 2009). Lonetti and colleagues also found that environmental enrichment ameliorated memory deficits and reduced anxiety-related behaviors in $\mathrm{MeCP} 2^{\text {tm1 Jae }} \mathrm{KO}$ females.

The effects of enriched environments have also been studied in mouse models of Fetal Alcohol Spectrum Disorders (FASDs). FASDs can occur in children whose mothers consume alcohol during pregnancy. Though the presentation of FASDs can vary in severity, behavioral manifestations in children generally include deficits in cognitive performance, executive functioning, and sensory-motor function (Jirikowic et al. 2008; Kodituwakku 2009). Rodent offspring whose mothers are exposed to ethanol display similar deficits. Attenuation of motor and learning deficits has been demonstrated in rodents (rats, mice) raised in or exposed to enriched conditions (Hannigan et al. 1993; Wainwright et al. 1993). Hannigan and colleagues found that rats exposed prenatally to alcohol and raised in impoverished conditions had locomotor gait dysmetrias indicative of ataxic gait. Environmental enrichment post-weaning ameliorated this atypical motor pattern and improved performance on a structured learning task. Similar improvements in spatial learning were found in prenatally ethanol-exposed mice (Wainwright et al. 1993). Despite these changes in behavioral function, support for neurological changes in prenatally 


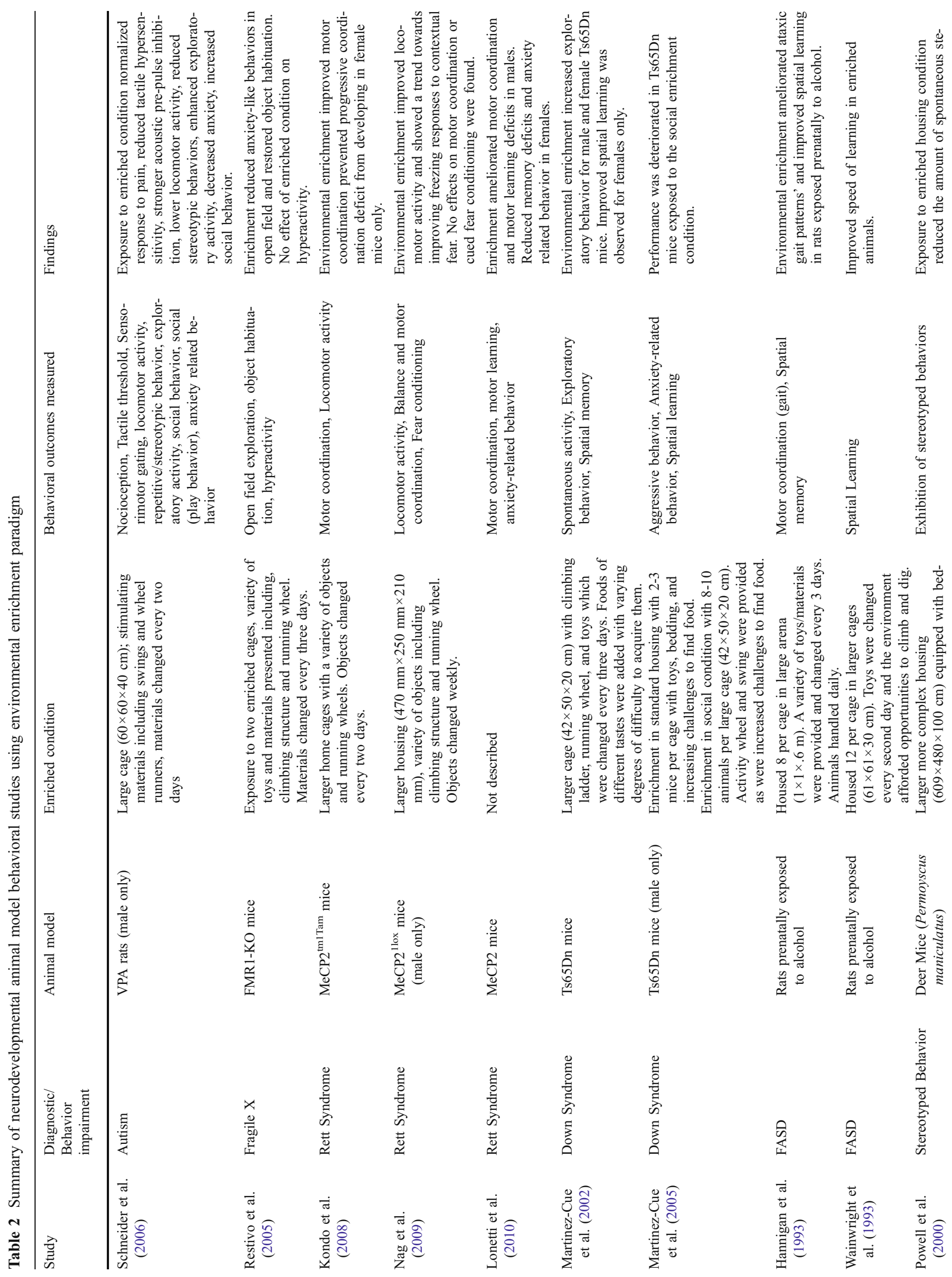


ethanol-exposed animals following environmental enrichment has not been documented (Berman et al. 1996; Parks et al. 2008; Wainwright et al. 1993).

Lastly, Down syndrome is a common genetic cause of mental retardation, and is often accompanied by behavioral disorders and attention deficits (Visootsak and Sherman 2007). A mouse model of Down syndrome (Ts65Dn mice) has been developed which replicates these impairments via a partial trisomy of murine chromosome 16 (MMU16). Female Ts65Dn mice exposed to enriched environmental conditions were found to demonstrate increased memory and spatial learning as measured using the Morris Water Maze (Martinez-Cue et al. 2002). Conversely, maleTs65Dn mice exposed to the same experimental condition showed regression in performance, suggesting that some environmental effects may be gender dependent.

Other work using the enriched environment paradigm has focused on specific symptoms of neurodevelopmental disorders as opposed to using animal models which mimic the disorder itself. For example, high rates of spontaneous and persistent stereotyped behaviors (jumping, backward flipping) can be observed in deer mice (Perromyscus maniculatus) housed in standard laboratory cages (Powell et al. 1999). Lewis and colleagues have studied this behavior extensively in this breed of rodents. Their findings indicate that mice housed in larger and more complex environments are less likely to engage in stereotyped behaviors compared to animals housed in standard conditions. The effects of this 'enrichment' were found both in early development and in adulthood. Further, mice exposed to the enriched environment in early development maintained the protective effects gained from the enriched condition even after they were placed in standard housing for an identical period of time (Powell et al. 2000). Subsequent studies examined the neurobiological basis for the effects of enriched environments using this breed of deer mice. Grouping mice based on whether they did or did not develop stereotyped behaviors, investigators found that mice exposed to enriched considitions that did not develop sterotyipies (non-stereotypic mice) exhibited significantly more neuronal metabolic activity (assessed using cytochrome oxidase histochemistry) in the motor cortex, enhanced dendritic spine densities in the motor cortex and the striatum, and enhanced levels of brain-derived neurotrophin factor (BDNF) in the striatum (Turner and Lewis 2003; Turner et al. 2003, 2002). While stereotypic enrichedcondition mice often showed gains above those housed in standard conditions, they failed to benefit from the enriched condition to the same extent as non-stereotypic enrichedcondition animals. These researchers suggest that enriched mice that display stereotypies may fail to engage with the environmental enrichment in meaningful ways, and therefore do not display the neurological changes (e.g. dendritic growth) associated with enrichment. 
Finally, there is evidence from primate research that prenatal stress can lead to behavioral deficits often observed in children with neurodevelopmental disorders, including sensory hypersensitivity, anxiety, impairments in social behavior, and alterations in the hypothalamic-pituitaryadrenal (HPA axis) (Schneider et al. 2008). Work by Laviola and colleagues has replicated these findings in rodents and produced evidence that exposure to enriched environments can reverse some of the effects of prenatal stress (Morley-Fletcher et al. 2003). In an experimental group of prenatally stressed rats, Laviola and colleagues found both neurological and behavioral improvements associated with engagement in an enriched environment, including increased social behavior and normalization of HPA axis reactivity (reduced peak and a return to baseline levels) (Laviola et al. 2004; Morley-Fletcher et al. 2003).

The findings reported here and summarized in Table 2 demonstrate that animal models of neurodevelopmental disorders may be useful in guiding understanding of the impact of interventions on behavior. Second, and more importantly, these studies indicate that a number of behavioral symptoms associated with neurodevelopmental disorders may be ameliorated or reduced with exposure to enriched environments, likely via mechanisms of experience dependent neural plasticity. Implications for the treatment of neurodevelopmental disorders in human populations need to be explored.

\section{Environmental controls: what is causing change?}

Since enriched environmental paradigms have varied, with some emphasizing a range of sensory and motor exploration opportunities while others emphasize socialization with other animals, it has raised the question, what aspect of the enriched environment are most salient in causing change? It has been well documented that exercise alone has considerable positive effects on the brain including improvements in learning and memory (for a review see van Praag 2009); it thus is feasible that increased motor activity may alone account for the global changes seen in the environmental enrichment paradigm. Thus far, however, this hypothesis has not proven true. For example, Percaccio and colleagues $(2005,2007)$ studied the effects of multiple environmental paradigms on rat's response to auditory tones. They found that auditory evoked responses were not altered by exercise or social stimulation, rather that rich auditory experiences themselves were the most effective in stimulating physiological plasticity of the auditory cortex. This suggests that for some sensory systems plasticity requires specific sensory input. Other studies have failed to show equivalent improvements in rodents exposed to wheel running only compared to rodents exposed to enriched environments, with those exposed to the enriched environment showing greater changes (Pang et al. 2006; Pawlowicz et al. 2009). Pang and colleagues (2006) suggest that while enhanced physical activity contributes to some of the beneficial effects of enrichment, sensory and cognitive stimulation are also essential components of this paradigm.

Renner and Rosenzweig (1986) examined the hypothesis that brain differences found in environmental enrichment are due to differences in social interaction or social play (wrestling \& chasing). These investigators compared grouphoused rats raised under standard conditions with grouphoused rats raised in an enriched condition and found no consistent pattern of differences in social interaction between the groups. These authors suggested that social interaction may have some direct or indirect (e.g. the activity of one animal attracts the attention of another) impact on the effects of environmental enrichment, but that it cannot account wholly for changes in neurological structure or function. Martinez-Cue and colleagues (2005) further suggest that social interaction may actually have negative effects on cognitive performance in certain animal models of dysfunction. These researchers found that Ts65Dn mice, an accepted animal model for Down syndrome, showed diminished cognitive performance and higher stress hormones when placed in enriched housing in large groups. These authors concluded that that excess social stimulation may actually disturb the emotional and behavioral components of the learning process thereby having an overall impact on cognitive function.

Extrapolating from these findings, the key drivers of neural plasticity found in environmental enrichment studies, while perhaps somewhat dependent on the type of neural dysfunction, appear to include the availability of multiple relevant sensory experiences coupled with novelty in the environment, and opportunities for engagement in challenging cognitive, sensory, and motor tasks. Exercise (in contrast to free motor exploration of the novel environment) and social stimulation potentially add value to the overall effects, at least in some paradigms, and the combined effects of sensory, motor, and cognitive stimulation may be synergistic rather than simply additive (Pawlowicz et al. 2009).

Further, neuroplastic changes tend to be functionally relevant. Animal studies of the auditory system have documented that neuroplastic changes result from both reward-associated effects and suppressive plasticity serving to reduce attention to non-target (distractor) inputs; neuroplasticity served to strengthen desired responses, and weaken those to distracters in the environment (Beitel et al. 2003). Studies in rehabilitation with humans indicate that structural changes secondary to stroke are functional, serving to reconnect regions of the motor cortex with the spinal cord (Ward 2005). These key features are important to keep in mind as we move on to consider how this body 
of knowledge can be used to inform the field of sensory integration intervention.

\section{Models of environmental enrichment informing Ayres Sensory Integration research paradigms}

It must be made clear that none of the rodent studies reviewed in this paper employed specific sensory integration methods. However, given the documented effectiveness of enriched environments in improving multiple areas of function in animal models of neurodevelopmental disorders there is an impetus to look at the clinical translation of this work into intervention for the human population. Due to the similarity between the essential features of enriched environments and SI treatment, it seems reasonable to consider that SI treatment has potential to be effective in the amelioration of some of the symptoms associated with neurodevelopmental conditions. Clearly evidence for this translation has yet to be solidified; the parallels drawn in the ensuing discussion are speculative. While therapists have been examining sensory integration intervention for years, this investigative field is fraught with challenges. Parham et al., (in press) emphasized that while over 70 articles have been published based on Ayres' work, intervention fidelity is a major concern. Failure of researchers to explicitly describe sensory interventions, report principles guiding these interventions, or include procedures to monitor intervention delivery has led to the publication of many articles whose results are generally inconclusive (Parham et al., in press). Randomized controlled trials have yet to be performed using SI intervention, leaving many professionals doubtful about its utility in the clinical field (National Autism Center 2009; Williames and Erdie-Lalena 2009). None-the-less, Parham and colleagues (2007) have offered guidelines that provide explicit descriptions of the content and procedures inherent in SI intervention, making examination of its core features relative to environmental enrichment feasible.

The term "Ayres Sensory Integration ${ }^{\circledR}$ " (Ayres SI) used here has been coined to represent the model of intervention that reflects the structural and process elements identified as core to sensory integration treatment. The structural and process elements have been formulated into a treatment fidelity instrument which has been tested for inter-rater reliably and internal consistency with strong markers for both $(\mathrm{ICC}=.99$, alpha $=.99)($ Parham et al. 2007). This tool is an important step in testing whether Ayres SI is truly effective.

At this point methodological issues need to be addressed to pursue a program of research in this area. Questions that need to be answered include: What are the most salient features of intervention (e.g. motor activity, environmental challenges, or sensory experiences)? Is there an ideal combination of features, or must all be present in order for treatment to be effective?; What is the role of the therapist and what is the level of variability in outcomes relative to therapist skill in intervention delivery?; What outcomes can we expect from Ayres SI treatment?; Who is Ayres SI therapy appropriate for and/or at what point in the lifespan should therapy occur?; and What is the optimal 'dosage' and duration of treatment? We will use the environmental enrichment literature described above to inform and focus on two of these questions: what are useful outcomes to target based on what has been shown in environmental enrichment and how best to address potential confounds. Other issues will also be considered.

Drawing from evidence from models of environmental enrichment, the following variables seem appropriate as potential outcomes for Ayres SI treatment:

- decreased anxiety

- decreased hypersensitivity to non-painful stimuli

- reduction/normalization of stress responses (e.g. salivary cortisol)

- increased social behavior and/or participation

- reduction in repetitive/stereotyped behaviors

- increased learning and memory (to be defined)

Exposure to enriched environments increased exploratory activity/behavior in animal models of autism, Fragile $\mathrm{X}$ syndrome, and Down syndrome. In addition, reductions in anxiety were displayed in the animal models of autism, Rett syndrome and Fragile $\mathrm{X}$ syndrome, and increased social behavior was noted in VPA-treated rats and rats exposed to prenatal stress. These findings suggest that when the animals are less anxious or fearful about their surroundings there is an increased motivation to engage with features of their environment. While this animal research cannot be directly applied to human populations, they provide data that must be seriously considered. If the outcome for these animal models of disability is reduced anxiety and possibly increased social participation following environmental enrichment, might these same outcomes be appropriate in examining the effectiveness of Ayres SI?

Reduced sensory hypersensitivity may be another outcome variable to consider with respect to Ayres SI. Sensory hypersensitivity has been linked to anxiety in children with ADHD and autism spectrum disorders (Reynolds and Lane 2009; Pfeiffer et al. 2005; Lane et al. 2010). Sensory hypersensitivity has also been linked to heightened levels of salivary cortisol, the hormonal stress by-product of the HPA axis, in children with ADHD (Reynolds et al. 2009). Reductions in hypersensitivity to non-painful sensory stimuli were noted in rat models of autism following environmental enrichment, and normalization of HPA axis reactivity was seen following environmental enrichment of rats exposed to prenatal stressors. 
Reduction in repetitive/stereotyped behaviors has been shown to be an outcome of enriched environments using a non-diagnostic animal model (Powell et al. 1999) and these behavioral changes have been associated with specific measures of neural plasticity (Turner and Lewis 2003; Turner et al. 2003, 2002). Smith and colleagues (2005) found similar behavioral results using SI treatment for children with pervasive developmental delay and mental retardation. In this study sensory integration intervention significantly reduced self-stimulatory and self-injurious behaviors suggesting that this treatment approach may be effective in reducing behaviors that interfere with the ability to participate in more functional activities.

Finally, animal models of conditions such as Alzheimers disease and Parkinson's disease suggest that environmental enrichment may be extremely beneficial for improving functional behaviors associated with enhanced learning and memory; these improvements are linked to neurogenesis of cells in the hippocampus. Such cognitive outcomes have not been examined to any extent in animal models of pediatric developmental disorders; the exception being an animal model of Rett syndrome where positive changes in procedural memory and motor learning have been documented following enriched conditions (Lonetti et al. 2010). These findings should provide impetus for further studies. However, investigating any potential link between Ayres Sensory Integration $\odot$ intervention and learning/memory changes will require careful identification of appropriate outcomes, and careful measurement of change. For example, will the child be expected to learn a new motor task (e.g. jumping jack), a new functional skill (e.g. shoe tying), or show improvements in an academic task such as reading or writing following a course of intervention? Is one outcome more likely than another? Quantifying such outcomes will present a challenge.

Research using animal models of enriched environment also provide us with insights as to what features of Ayres SI may be most salient. As noted previously, the key features in the enriched environment paradigms appear to be the presence of:

- multiple sensory experiences

- novelty in the environment

- active engagement in challenging cognitive, sensory, and motor tasks

Therefore, in terms of treatment, it should be the primary goal of the therapist to provide or facilitate multiple sensory experiences, set up the therapeutic environment to facilitate these experiences and exploration, introduce novelty into the environment and/or the activity, and consistently enhance complexity to instigate cognitive, motor and sensory challenges. In broad terms these requirements are very consistent with the structure and process features defined in the treatment fidelity tool described above.
In addition, control over potentially confounding variables will need to be examined. Confounding factors will include variability in physical activity and social engagement, and therapist variability in implementing these key features. To control for the variable of physical activity there are at least two options; 1) children engaging in treatment can wear accelerometers and activity variables can be accounted for statistically; or 2) research paradigms can be set up in which Ayres SI is compared to an exercise-only control condition similar to the animal model. A similar methodology could be employed to deal with the variable of social engagement where children can engage in traditional Ayres SI treatment (one on one with a therapist) or in small or large groups.

Variability of the therapist, or the therapist's role, certainly presents a greater challenge. Viewed from a very simple perspective however, the first step may be to compare an Ayres SI trained therapist to the provision of free access to a therapeutic environment with therapist supervision for safety. This second therapist would parallel the role of the researcher in the animal enriched environment model, which is to simply to set up the condition and make occasional changes. Parham et al., (in press) have demonstrated that independent raters can accurately distinguish between a trained therapist doing SI intervention and a caregiver supervising a child in a therapy room. If the importance of a trained therapist is substantiated, variability in outcomes based on therapists level of experience, skill, or involvement would be the next step in the research process.

In considering the question for whom is SI therapy appropriate and/or at what point in the lifespan should therapy occur, information may also be gained from the animal literature reviewed here. Outcome variables discussed above indicate that Ayres SI may be beneficial for individuals presenting with anxiety, hypersensitivity, repetitive/stereotyped behaviors or heightened levels of stress. Additionally, individuals with deficits in social behavior or social participation may show gains with this type of treatment (potentially due to reductions in anxiety, stress, and hypersensitivity). Due to implications for learning and memory, Ayres SI may also be important to consider for individuals with learning disabilities, developmental dyspraxia, or recovering from an acute neurological insult (brain injury or stroke). Further, mounting research suggests that environmental enrichment may be both preventative and restorative and its benefits can occur not only in pre-pubertal years but well into adulthood. In fact, this research raises the question of why Ayres SI has predominantly been considered for children with neurodevelopmental disorders, when evidence suggests that exposure to this type of treatment may be beneficial across the lifespan. Assessment of Ayres SI intervention across the lifespan will be a big task, and represents an extension of the current theoretical basis, but worth considering in future studies. 
Answering the final question, that of dosage, is one that perhaps does not have a foundation in animal literature. The life span and developmental course of rodents differs greatly from humans, and a dosage plan for rodents does not translate to a dosage plan for children. However, it is interesting to note that in many of the environmental enrichments studies animals had free access to their enriched environments, meaning they could engage daily. Alternative designs involved several exposures a week. The questions related to minutes/session, times/week and overall duration for effectiveness of Ayres SI treatment will come from careful examination of experiences with children in the clinical setting, and careful study design.

\section{Conclusion}

The literature presented suggests that principles from basic science (primarily rodent) research, such as the environmental enrichment paradigm, may be used to inform research on interventions used to treat children with neurodevelopmental disorders. A review of studies using the environmental enrichment paradigm over the past 50 years suggests that engaging in an opportunity-rich environment with novel sensory, motor and cognitive challenges can initiate lasting and functional changes in the brain. These changes appear to correspond to a reduction in behaviors such as anxiety and hypersensitivity and an enhancement in social interaction, learning, and memory. Based on the overlapping essential features of the enriched environment animal model and Ayres Sensory Integration ${ }^{\circledR}$, there is reason to deduce that this type of intervention may be appropriate for children with neurodevelopmental disorders paralleling diagnostic models used in the environmental enrichment studies. The challenge is to design rigorous studies that investigate this possibility.

The translation of animal enrichment models to human studies of Ayres SI requires consideration of a couple of issues. First, comparison groups in studies of enriched environments often involve low-stimulation or impoverished conditions; the same cannot be said for children beginning a course of sensory integration treatment. Most children in developing countries are at no loss for stimulation or opportunities to engage in a stimulating environment. Why then, would we expect therapy aimed at providing these opportunities to be effective? It appears that many children with neurodevelopmental disorders such as autism, while exposed to enriched home and school environments, lack the ability to effectively engage in those environments or to participate with objects and people in a meaningful way. Several animal studies have, in fact, shown that control animals benefit more from enriched environments than animals with impaired behavior, cognitive or social functioning (Berman et al. 1996; Martinez-Cue et al. 2002; Dierssen et al. 2003; Turner et al. 2002; Turner and Lewis 2003; Turner et al. 2003). This would suggest that it is not simply exposure to the environment but engagement with the environment (e.g. physical, social) that is essential to bring about neurological changes. Children with cognitive, behavioral, social, sensory, or motor deficits may need the assistance of a trained therapist to maximize their ability to engage in complex environments. Neuronal plasticity involved in learning appears to be highly dependent upon sensory inputs that are meaningful, in that they reliably predict behaviorally reinforcing events (Blake et al. 2006; Polley et al. 2006; Seitz and Wantanabe 2005).Therefore, meaningful engagement in sensory integration treatment may be accomplished, in part, through enhancing motivation and attention to task (Kleim and Jones 2008). As such, for the use of Ayres SI with humans, it will not be a simple matter of creating a generically enriched environment, but instead one that addresses the specific needs of the child and capitalizes on the therapeutic alliance, as has been suggested by Parham and colleagues (2007, in press). The unique feature of Ayres SI relative to the child's typical environment therefore, is the therapist working to create and provide sensory opportunities specific to the needs of the child, and to facilitate interaction and motivation, support optimal arousal, and work beyond the therapy session with families and teachers (Parham et al., in press). The therapist in this case serves as the scientist might, to tease out the specific aspects of the enrichment paradigm that best meets the needs of the child.

The second issue to consider is how Ayres SI treatment can be conceptualized and studied within the broader field of rehabilitation of neurodevelopmental disorders. Sensory integration theory suggests that adequate processing of sensory information provides a foundation for more advanced adaptive responses and participation in cognitive and motor tasks (Ayres 1998; Baranek 2002). From this perspective, SI treatment emphasizes processing of sensation as the foundation for the higher level cognitive abilities to organize, integrate and utilize this information in environmental interaction. The input focus is sensory-based, the output from the child is active environmental interaction. In contrast, explicit skill-based training is focused on the skill itself. The input is skill repetition and practice, the output is a specific skill. Skill-based programs have been be classified as 'top-down' because of the emphasis on the cognitive drive to produce the desired response (Marshall 2009). From a rehabilitation perspective, it might be argued that a topdown approach would be more appropriate for treating deficits associated with neurodevelopmental disorders. In fact, both human and animal research has shown enhanced neural plasticity in specific brain regions associated with repetitive task training (e.g. reaching task). While factors such as intensity, time and salience matter when it comes to these training paradigms, there is a plethora of research to 
show that repetition of specific tasks can stimulate both behavioral changes (e.g. ability to perform specific behavior) and changes in neuronal connectivity (for a review see Kleim and Jones 2008). There is still some question, however, if isolated task training can effectively generalize to functional changes in performance. A meta-analysis by French and colleagues (2010) suggests that repetitive task training for lower limbs may be effective in bringing about functional activity gain in post-stroke patients, while repetitive training of upper limbs is not. Further research certainly is warranted to explore these methods of rehabilitation; and additional studies specific to functional outcomes in children with neurodevelopmental disabilities will be imperative. It may be worth considering that a combined approach of environmental enrichment (or Ayres SI) and repetitive task training may be the most efficacious for stimulating neural plasticity and long lasting functional changes.

In addition to differing from approaches that are skill based, Ayres SI differs from what are termed 'sensory stimulation' approaches to intervention. Such sensory stimulation interventions would clearly fall into what has been termed 'bottom-up' approaches; approaches that do not require the conscious effort or engagement of the client (Blake et al. 2006; Marshall 2009). Ayres SI does not fall easily into either bottom-up or top-down categories; there is clear emphasis on sensory input as key, but there is equal emphasis on interaction with both social and physical environments, thus requiring higher level processing and engagement. Importantly for this paper, neither 'top-down' nor sensory stimulation approaches share the essential features delineated earlier that link environmental enrichment and sensory integration. Thus, while there may be some efficacy in the use of other approaches, discussion of these topics is outside the realm of this paper.

Specific to pediatric neurodevelopmental disorders, behavioral intervention models (e.g. applied behavioral analysis $[\mathrm{ABA}]$ ) have emerged as the most used and scientifically validated techniques for treating children with autism. However, while changes in specific skill acquisition have been demonstrated, evidence continues to be needed regarding generalizability of gained skills, both across tasks and across environments. Learning skills through reward and repetition has some grounding in the research reviewed here, but the approach used with ABA is quite different from that seen in the animal studies. Whether actual neuroplastic changes take place, and can be maintained, remains unclear. While proponents of ABA advocate for the intensive and sole use of this approach (Foxx 2008), there is evidence to suggest that meaningful, lasting, and functional changes in the brain can be achieved through use of other sensory-motor strategies. It is certainly too soon to discount the effects of these types of treatments when research on the etiology of the neurodevelopmental disorders and factors impacting long term outcomes are still being considered. Future work in the area of basic science may help to elucidate the salient aspects of therapeutic approaches which can be targeted to address specific symptoms or behavioral manifestations of neurodevelopmental disorders. It is certain that there is not one treatment (pharmacological or rehabilitative) that will address all of the symptoms of all conditions which affect the development of children. The role of translational research will be imperative in using knowledge gained in basic science and applying it to human populations. As noted by Kleim and Jones (2008); “...findings from animal models of neurological disorders do not automatically translate to specific recommendations for the clinic. Rather, our role is to study neurobiological phenomenon related to functional recovery and to identify fundamental principles that may help to guide the optimization of rehabilitation" (p. S225).

\section{References}

American Psychiatric Association. Diagnostic and statistical manual of mental disorders: DSM-IV-TR. Washington: American Psychiatric Association, Inc.; 2000.

Ayres AJ. Sensory integration and the child. 13th ed. Los Angeles: Western Psychological Services; 1998.

Baranek GT. Efficacy of sensory and motor interventions for children with autism. J Autism Dev Dis. 2002;32:397-422.

Baranek GT, David FJ, Poe MD, Stone WL, Watson LR. Sensory experiences questionnaire: discriminating sensory features in young children with autism, developmental delays, and typical development. J Child Psychol Psych. 2006;47:591.

Beitel R, Schreiner C, Cheung S, Wang X, Merzenich M. Rewarddependent plasticity in the primary auditory cortex of adult monkeys trained to discriminate temporally modulated signals. Proc Natl Acad Sci USA. 2003;100:11070-5.

Berman RF, Hannigan JH, Sperry MA, Zajac CS. Prenatal alcohol exposure and the effects of environmental enrichment on hippocampal dendritic spine density. Alcohol. 1996;13(2):209-16.

Bezzi P, Gunderson V, Galbete JL, Seifert G, Steinhauser C, PIlati E, et al. Astrocytes contain a vesicular compartment that is competent for regulated exocytosis of glutamate. Nat Neurosci. 2004;7:613-20.

Blake DT, Heiser MA, Caywood M, Merzenich MM. Experiencedependent adult cortical plasticity requires cognitive association between sensation and reward. Neuron. 2006;52:371-81.

Bohm HV, Stewart MG. Brief report: On the concordance percentages for autistic spectrum disorder of twins. J Autism Dev Disord. 2009;39:806-8.

Bose M, Munoz-Llancao P, Roychowhury S, Nichols JA, Jakkamsetti V, Porter B, et al. Effect of the environment on the dendritic morphology of the rat auditory cortex. Synapse. 2010;64:97-110.

Breedlove MS, Rosenzweig MR, Watson NV, editors. Biological psychology: an introduction to behavioral, cognitive and clinical neuroscience. 5th ed. Sunderland, MA: Sinauer Acssociates, Inc.; 2007.

Brown J, Cooper-Kuhn CM, Kempermann G, Van Praag H, Winkler J, Gage $\mathrm{FH}$, et al. Enriched environment and physical activity stimulate hippocampal but not olfactory bulb neurogenesis. Eur J Neurosci. 2003;17:2042-6.

Bruel-Jungerman E, Laroche S, Rampon C. New neurons in the dentate gyrus are involved in the expression of enhanced long- 
term memory following environmental enrichment. Eur J Neurosci. 2005;21:513-21.

Christodoulou J, Williamson SL. Rett syndrome: New clinical and molecular insights. Eur J Hum Genet. 2006;14:896-903.

Connor JR, Wang EC, Diamond MC. Increased length of terminal dendritic segments in old adult rats' somatosensory cortex: An environmentally induced response. Exp Neurol. 1982;78:466-70.

Dierssen M, Benavides-Piccione R, Martinez-Cue C, Estivill X, Florez J, Elston GN, et al. Alterations of neocortical pyramidal cell phenotype in the Ts65Dn mouse model of down syndrome: Effects of environmental enrichment. Cereb Cortex. 2003;13:7587-764.

Dong WK, Greenough WT. Plasticity of nonneuronal brain tissue: Roles in developmental disorders. Men Retard Dev D R. 2004;10:85-90.

Faherty CJ, Kerley D, Smeyne RJ. A golgi-cox morphological analysis of neuronal changes induced by environmental enrichment. Dev Brain Res. 2003;141:55-61.

Faherty CJ, Raviie Shepherd K, Herasimtschuk A, Smeyne RJ. Environmental enrichment in adulthood eliminates neuronal death in experimental parkinsonism. Mol Brain Res. 2005;134:170-9.

Fazlioglu Y, Baran G. A sensory integration therapy program on sensory problems for children with autism. Percept Mot Skills. 2008;106:415-22.

Floeter MK, Greenough WT. Cerebellar plasticity: modification of purkinje cell structure by differential rearing in monkeys. Science. 1979;206:227-9.

Folstein SE, Rosen-Sheidley B. Genetics of autism: complex aetiology for a heterogeneous disorder. Nat Rev Genet. 2001;2:943-55.

Foxx RM. Applied behavior analysis treatment in autism: the state of the art. Child Adolesc Psychiatr Clin N Am. 2008; 17:821-34.

French B, Thomas L, Leathley M, Sutton C, McAdam J, Forster A, et al. Does repetitive task training improve functional activity after stroke? A cochrane systematic review and meta-analysis. J Rehabil Med. 2010;42:9-15.

Green VA, Pituch KA, Itchon J, Choi A, O'Reilly M, Sigafoos J. Internet survey of treatments used by parents of children with autism. Res Dev Disabil. 2006;27:70.

Greenough WT, Volkmar FR. Pattern of dendritic branching in occipital cortex of rats reared in complex environments. Exp Neurol. 1973;40:491-504.

Greenough WT, McDonald JW, Parnisari RM, Camel JE. Environmental conditions modulate degeneration and new dendrite growth in cerebellum of senescent rats. Brain Res. 1986;380:136-43.

Hannigan JH, Berman RF, Zajac CS. Environmental enrichment and the behavioral effects of prenatal exposure to alcohol in rats. Neurotoxicol Teratol. 1993;15(4):261-6.

Hebb DO. The effects of early experience on problem solving and maturity. Amer Psychol. 1947;2:306.

Herring A, Ambree O, Tomm M, Habermann H, Sachser N, Paulus W, et al. Environmental enrichment enhances cellular plasticity in transgenic mice with Alzheimer-like pathology. Exp Neurol. 2009;216:184-92.

Jirikowic T, Olson HC, Kartin D. Sensory processing, school performance, and adaptive behavior of young school-age children with fetal alcohol spectrum disorders. Phys Occup Ther Pediatr. 2008;28(2):117-36.

Kempermann G, Gage FH. Neurogenesis in the adult hippocampus. Novartis Found Symp. 2000;231:220-35.

Kleim JA, Jones TA. Principles of experience-dependent neural plasticity: implications for rehabilitation after brain damage. J Speech Lang Hear R. 2008;51:S225-39.
Kodituwakku PW. Neurocognitive profile in children with fetal alcohol spectrum disorders. Dev Disabil Res Rev. 2009;15 (3):218-24.

Kondo M, Gray LJ, Pelka GJ, Christodoulou J, Tam PPL, Hannan AJ. Environmental enrichment ameliorates a motor coordination deficit in a mouse model of rett syndrome- Mecp2 gene dosage effects and BDNF expression. Eur J Neurosci. 2008;27:3342-50.

Krech D, Rosenzweig MR, Bennett EL. Effects of environmental complexity and training on brain chemistry. J Comp Physiol Psychol. 1960;53:509-19.

Lane, S. J., Reynolds, S., \& Thacker, L. (2010). Sensory overresponsivity and ADHD: Differentiating using electrodermal responses, cortisol, and anxiety. Front Integr Neurosci.

Laviola G, Rea M, Morley-Fletcher S, Di Carlo S, Bacosi A, De Simone R, et al. Beneficial effects of enriched environment on adolescent rats from stressed pregnancies. Eur J Neurosci. 2004;20:1655-64.

Leekman SR, Nieto C, Libby SJ, Wing L, Gould J. Describing the sensory abnormalities of children and adults with autism. J Autism Dev Disord. 2006. doi:10.1007/s10803-006-0218-7.

Leggio MG, Mandolesi L, Federico F, Spirito F, Ricci B, Gelfo F, et al. Environmental enrichment promotes improved spatial abilities and enhanced dendritic growth in the rat. Behav Brain Res. 2005; 163:78-90.

Lonetti G, Angelucci A, Morando L, Boggio EM, Giustetto M, Pizzorusso T. Early environmental enrichment moderates the behavioral and synaptic phenotype of MeCP2 null mice. Biol Psychiatry. 2010;67:657-65.

Marshall RS. Rehabilitation approaches to hemineglect. Neurologist. 2009; 15:185-92.

Martinez-Cue C, Baamonde C, Lumbreras M, Paz J, Davisson MT, Schmidt C, et al. Differential effects of environmental enrichment on behavior and learning of male and female Ts65Dn mice, a model for Down syndrome. Behav Brain Res. 2002;134:185200.

Martinez-Cue C, Rueda N, Garcia E, Davisson MT, Schmidt C, Florez J. Behavioral, cognitive and biochemical responses to different environmental conditions in male Ts65Dn mice, a model of Down syndrome. Behav Brain Res. 2005;163:174-85.

Miller LJ, Coll JR, Schoen SA. A randomized controlled pilot study of the effectiveness of occupational therapy for children with sensory modulation disorder. Am J Occup Ther. 2007;61:228-38.

Morley-Fletcher S, Rea M, Maccari S, Laviola G. Environmental enrichment during adolescence reverses the effects of prenatal stress on play behaviour and HPA axis reactivity in rats. Eur J Neurosci. 2003;18:3367-74.

Nag N, Moriuchi JM, Peitzman CGK, Ward BC, Kolodny NH, Berger-Sweeney JE. Environmental enrichment alters locomotor behaviour and ventricular volume in Mecp21lox mice. Behav Brain Res. 2009;196:44-8.

National Autism Center. (2009). National standards report. Retrieved March/31, 2010, from http://www.nationalautismcenter.org/pdf/ NAC\%20Standards\%20Report.pdf.

Nithianantharajah J, Hannan AJ. Enriched environments, experience dependent plasticity and disorders of the nervous system. Nat Rev Neurosci. 2006;7:697-709.

Okuda H, Tatsumi K, Makinodan M, Yamauchi T, Wanaka A. Environmental enrichment stimulates progenitor cell proliferation in the amygdala. J Neurosci Res. 2009;87:3546-53.

Pang TY, Stam NC, Nithianantharajah J, Howard ML, Hannan AJ. Differential effects of voluntary physical exercise on behavioral and brain-derived neurotrophic factor expression deficits in Huntington's disease transgenic mice. Neuroscience. 2006;141:569-84.

Parham LD, Cohn ES, Spitzer S, Koomar JA, Miller LJ, Burke JP, et al. Fidelity in sensory integration intervention research. Am J Occup Ther. 2007;61:216-27. 
Parham, L. D., Smith Roley, S., May-Benson, T. A., Koomar, J., BrettGreen, B., Burke, J. P., et al. (in press). Development of a fidelity measure for research on effectiveness of ayres sensory integration intervention. Am J Occup Ther.

Parks EA, McMechan AP, Hannigan JH, Berman RF. Environmental enrichment alters neurotrophin levels after fetal alcohol exposure in rats. Alcohol Clin Exp Res. 2008;32(10):1741-1751.

Pawlowicz A, Demner A, Lewis MH. Effects of access to voluntary wheel running on the development of stereotypy. Behav Process. 2009. doi:10.1016/j.beproc.2009.11.008.

Percaccio CR, Engineer ND, Pruette AL, Pandya PK, Moucha R, Rathbun DL, et al. Environmental enrichment increases pairedpulse depression in rat auditory cortex. J Neurophysiol. 2005;9:3590-600.

Percaccio CR, Pruette AL, Mistry ST, Chen YH, Kilgard MP. Sensory experience determines enrichment-induced plasticity in rat auditory cortex. Brain Res. 2007;1174:76-91.

Pfeiffer B, Kinnealey M, Reed C, Herzberg G. Sensory modulation and affective disorders in children and adolescents with asperger's disorder. Am J Occup Ther. 2005;59:335-45.

Polley DB, Steinberg EE, Merzenich MM. Perceptual learning directs auditory cortical map reorganization through top-down influences. J Neurosci. 2006;26:4970-82.

Powell SB, Newman HA, Pendergast J, Lewis MH. A rodent model of spontaneous stereotypy: Initial characterization of developmental, environmental, and neurobiological factors. Physiol Behav. 1999;66:355-63.

Powell SB, Newman HA, McDonald TA, Bugenhagen P, Lewis MH. Development of spontaneous stereotyped behavior in deer mice: Effects of early and late exposure to a more complex environment. Dev Psychobiol. 2000;37:100-8.

Qian J, Zhou D, Pan F, Liu C, Wang Y. Effect of environmental enrichment on fearful behavior and gastrin-releasing peptide receptor expression in the amygdala of prenatal stressed rats. $\mathrm{J}$ Neurosci Res. 2008;86:3011-7.

Renner MJ, Rosenzweig MR. Social interactions among rats housed in grouped and enriched conditions. Dev Psychobiol. 1986;19:303-13.

Restivo L, Ferrari F, Passino E, Sgobio C, Bock J, Oostra BA, et al. Enriched environment promotes behavioral and morphological recovery in a mouse model for the fragile X syndrome. PNAS. 2005; 102:11557-62.

Reynolds S, Lane SJ. Sensory over-responsivity and anxiety in children with ADHD. Am J Occup Ther. 2009;63:443-0.

Reynolds S, Lane SJ, Gennings C. The moderating role of sensory overresponsivity in HPA activity. J Atten Disord. 2009. doi:10.1177/1087054708329906.

Rosenzweig MR, Krech D, Bennett EL, Diamond MC. Effects of environmental complexity and training on brain chemistry and anatomy: a replication and extension. J Comp Physiol Psychol. 1962;55:429-37.

Santangelo SL, Tsatsanis K. What is known about autism: genes, brain, and behavior. Am J Pharmacogenomic. 2005;5:71.
Savioz A, Leuba G, Vallet PG, Walzer C. Contribution of neural networks to alzheimer disease's progression. Brain Res Bull. 2009;80:309-14.

Schaaf RC, Nightlinger KM. Occupational therapy using a sensory integrative approach: a case of effectiveness. Am J Occup Ther. 2007;61:239-46.

Schneider T, Przewlocki R. Behavioral alterations in rats prenatally exposed to valproic acid: animal model of autism. Neuropsychopharmacol. 2005;30:80-9.

Schneider T, Turczak J, Przewlocki R. Environmental enrichment reverses behavioral alterations in rats prenatally exposed to valproic acid: issues for a therapeutic approach in autism. Neuropsychopharmacol. 2006;31:36-46.

Schneider ML, Moore CF, Gajewski LL, Larson JA, Roberts AD, Converse AK, et al. Sensory processing disorder in a primate model: Evidence from a longitudinal study of prenatal alcohol and prenatal stress effects. Child Dev. 2008;79:100-13.

Seitz A, Wantanabe T. A unified model for perceptual learning. Trends Cogn Sci. 2005;9:329-34.

Smith SA, Press B, Koenig KP, Kinnealey M. Effects of sensory integration intervention on self-stimulating and self-injurious behaviors. Am J Occup Ther. 2005;59:418-25.

Stearns NA, Schaevitz LR, Bowling H, Nag N, Berger UV, BergerSweeney J. Behavioral and anatomical abnormalities in MECP2 mutant mice: a model for rett syndrome. Neuroscience. 2007; 146:907-21.

Tomchek SD, Dunn W. Sensory processing in children with and without autism: a comparative study using the short sensory profile. Am J Occup Ther. 2007;61:190-200.

Turner AM, Greenough WT. Differential rearing effects on rat visual cortex synapses. Brain Res. 1985;329:195-203.

Turner CA, Lewis MH. Environmental enrichment: Effects on stereotyped behavior and neurotrophin levels. Physiol Behav. 2003;80:259-66.

Turner CA, Yang MC, Lewis MH. Environmental enrichment: effects on stereotyped behavior and regional neuronal metabolic activity. Brain Res. 2002;938:15-21.

Turner CA, Lewis MH, King MA. Environmental enrichment: effects on stereotyped behavior and dendritic morphology. Dev Psychobiol. 2003;43:20-7.

van Praag H. Exercise and the brain: Something to chew on. Trends Neurosci. 2009;32:283-90.

Visootsak J, Sherman S. Neuropsychiatric and behavioral aspects of trisomy 21. Curr Psychiatry Rep. 2007;9:135-40.

Wainwright PE, Levesque S, Krempulec L, Bulman-Fleming B, McCutcheon D. Effects of environmental enrichment on cortical depth and Morris-maze performance in B6D2F2 mice exposed prenatally to ethanol. Neurotoxicol Teratol. 1993;15(1):11-20.

Ward NS. Plasticity and the functional reorganization of the human brain. Int J Psychophys. 2005;58:158-61.

Williames LD, Erdie-Lalena CR. Complementary, holistic, and integrative medicine: sensory integration. Pediatr Rev. 2009;30(12):e91-3. 